\title{
MMP-Activated Fluorescence Imaging Detects Early Joint Inflammation in Collagen-Antibody-Induced Arthritis in CC-Chemokine Receptor-2-Null Mice, In-Vivo
}

\author{
Jessica M. Ibarra, ${ }^{1}$ Fabio Jimenez, ${ }^{1,2}$ Hernan G. Martinez, ${ }^{1,2}$ \\ Kassandra Clark, ${ }^{1,2}$ and Seema S. Ahuja ${ }^{1,2}$ \\ ${ }^{1}$ Department of Medicine, University of Texas Health Science Center at San Antonio, San Antonio, TX 78229-3900, USA \\ ${ }^{2}$ Veterans Administration Center for Research on AIDS and HIV-1 Infection and South Texas Veterans Health Care System, \\ Audie L. Murphy Division, San Antonio, TX, USA
}

Correspondence should be addressed to Seema S. Ahuja, ahuja@uthscsa.edu

Received 8 February 2011; Revised 12 March 2011; Accepted 18 March 2011

Academic Editor: Bernhard Rintelen

Copyright ( 2011 Jessica M. Ibarra et al. This is an open access article distributed under the Creative Commons Attribution License, which permits unrestricted use, distribution, and reproduction in any medium, provided the original work is properly cited.

\begin{abstract}
The Standard measures of experimental arthritis fail to detect, visualize, and quantify early inflammation and disease activity. Here, we describe the use of an injectable MMP-activated fluorescence agent for in vivo quantification of acute inflammation produced by collagen-antibody-induced arthritis (CAIA) in CC chemokine receptor-2 (Ccr2 $\left.{ }^{-/-}\right)$null mice. Although Ccr2 ${ }^{-/-} \mathrm{DBA} 1 / \mathrm{J}$ mice were highly susceptible to and rapidly developed CAIA, the standard clinical assessment of fore or hind paw thicknesses was unable to detect significant acute inflammatory changes (days 3-10). Remarkably, noninvasive, in situ, MMP-activatable fluorescent imaging of $\mathrm{Ccr} 2^{-/-}$DBA1/J mice with CAIA displayed acute joint pathology in advance of clinically measurable acute inflammation (days 5,7 , and 10). These results were confirmed by the histology of ankle joints, which showed significant inflammation, bone loss, and synovial hyperplasia, compared to control mice at postimmunization day 5. The MMP-mediated fluorescence technique holds tremendous implications for quantifiable examination of arthritis disease activity of acute joint inflammation.
\end{abstract}

\section{Introduction}

Rheumatoid arthritis (RA) is a chronic inflammatory joint disease with a substantial morbidity and mortality that affects approximately $1 \%$ of the US population $[1,2]$. Clinically, RA manifests as unrelenting pain, stiffness, progressive joint destruction, and functional disability, in addition to creating a huge burden for caregivers and impairing productivity [3]. Although the etiology of RA is not completely delineated, studies using animal models of arthritis led to the development of novel biological agents for the treatments of arthritis $[4,5]$. However, treatment success is limited by early diagnosis and early therapeutic interventions. What is more, by the time the signs of arthritis appear in the models of RA, the disease well passes the acute phase of the disease. To complicate things further the classical measures of disease activity (clinical scoring and alterations to paws) rely heavily on subjective, nonclinical measures of inflammatory changes (paw thickness and joint histology) and fail to impart acute quantitative measures of RA disease progression.

Recently, we described a protective role for CCR2 against collagen-induced arthritis (CIA) in mice [6, 7]. CCR2-null mice $\left(\mathrm{Crr} 2^{-/-}\right)$displayed greater incidence and severity of experimental arthritis compared to wild-type controls [6]. $\mathrm{Ccr}^{-/-}$mice developed extensive joint inflammation and histopathological features of reminiscent of RA with an enhanced CCR2-independent recruitment of neutrophils and monocytes/macrophages. During inflammation, CCR2 is expressed on the surface of monocytes, macrophages, $\mathrm{T}$ and $\mathrm{B}$ lymphocytes, natural killer cells, and dendritic cells to direct CCR2-mediated chemotaxis [8]. Coincident with the accumulation of inflammatory cells, the expression of matrix metalloproteinases (MMPs) also increases in early stages of arthritis $[9,10]$. In the present study, we hypothesized that 
MMP-activatable fluorescent in vivo imaging could serve as a marker of acute inflammation and early arthritis of CAIA in $\mathrm{Ccr}^{-/-}$mice. Our findings demonstrating the utility of an MMP-activated fluorescence agent not only provided a sensitive method for monitoring and imaging clinical synovitis, but also afforded us the ability to quantify inflammation prior to visible synovitis.

From our findings, we propose an alternative method for monitoring disease activity in RA disease models via targeted fluorescent imaging of protease activity. Compared to the standard measures of disease progression, fluorescence imaging quickly discriminated early inflammation and was more sensitive than the standard clinical measures of acute inflammation in experimental arthritis.

\section{Materials and Methods}

2.1. Mice. Mice were housed under a pathogen-free environment at the University of Texas Health Science Center at San Antonio, Tex, USA and procedures were performed according to the protocol approved by the Institutional Animal Care and Use Committee. Male Ccr2 ${ }^{-/-}$DBA1/J mice (6-8 weeks old) were developed as previously described [6].

2.2. Collagen-Antibody-Induced Arthritis (CAIA) Immunization. To examine early inflammation in CAIA, mice were injected with the collagen type II antibody, previously described by Quinones et al. [6]. Briefly, on day 0 mice received an intravenous injection of an antibody cocktail $(4 \mathrm{mg})$ consisting of four mouse antibodies to collagen type II (Chemicon International, Temecula, Calif, USA) at the base of the tail. On day 3, mice received a single intraperitoneal injection of $25 \mu \mathrm{g}$ lipopolysaccharide (LPS; Escherichia coli, serotype O55:B5; Sigma, St. Louis, MO) in $0.9 \%$ sterile saline.

2.3. In Vivo Fluorescent Imaging and Quantification. Twentyfour hours prior to imaging, CAIA/LPS-treated $\mathrm{Ccr}^{-/-}$ DBA1/J mice $(n=3)$ and control mice $(n=2)$ received an intramuscular injection of ketamine hydrochloride $(10 \mathrm{mg} / \mathrm{kg})$ to immobilize the mice. Immediately, the mice received one intravenous injection $(20 \mathrm{nmol} / \mu \mathrm{L})$ of an MMPactivatable (excitation: $680 \mathrm{~nm}$; emission: $700 \mathrm{~nm}$ ) fluorescent in vivo imaging agent (MMPsense 680, VisEn Medical, Woburn, Mass, USA) that remained optically silent in an unactivated state. Protease activation produced fluorescence (inflammatory-disease-state-activated, in situ). Control mice $(n=2)$ received phosphate-buffered-saline (PBS). This procedure was repeated on days 7 and 10. Fluorescence imaging was performed (excitation: $675 \mathrm{~nm}$; emission: $720 \mathrm{~nm}$ ) using the IVIS spectrum imaging platform (Caliper Life Sciences, Hopkinton, Mass, USA) with an excitation of $675 \mathrm{~nm}$ and an emission of $720 \mathrm{~nm}$ wavelengths in epi-illumination and transillumination modes in vivo. Images were captured using a CCD camera. Macrophage and neutrophil recruitment was quantified by measuring the fluorescence intensity (photons/second) produced by MMPsense 680 activation at the paw inflammation.
2.4. Clinical Assessment. The severity of arthritis was scored based on clinical scoring of the hind and fore paws, as previously described [6]. Briefly, the joint swelling was assigned a clinical score graded on the severity where swelling/erythema of hind and fore paws was graded from 0 (no swelling or erythema) to grade 4 (severe swelling with joint rigidity or deformity). The final clinical score (severity) was reported as the mean joint involvement from the hind and fore paws.

2.5. Histopathology. To determine the extent of disease destruction, the ankle joints were collected from CAIA/LPStreated and control mice, the skin removed, washed in PBS, and joints fixed overnight in paraformaldehyde (4\% in PBS, pH 7.2). The bone was decalcified in 10\% EDTA and followed by paraffin tissue embedding, serial sectioning $(5 \mu \mathrm{m})$, and stained with hematoxylin and eosin (H\&E). Paraformaldehyde, EDTA, hemotoxylin, and eosin were obtained from Sigma, St. Louis, Mo, USA.

2.6. Statistical Analyses. Data are presented as mean \pm SEM. Groups were analyzed using Stata (StataCorp, College Station, TX) with statistical significance at $P \leq .05$. Nonparametric statistical analyses with Spearman correlation for nonparametric data (paw thickness and photons/second) were performed with the Bonferroni correction.

\section{Results}

3.1. Clinical Assessment of CAIA. The development of acute arthritis in CAIA is dependent on the innate immunity, and the disease is primarily mediated by neutrophils and macrophages; therefore, we examined the onset of disease very early (days 3-10) by standard measures of clinical assessment. The susceptibility to arthritis, as represented by the clinical score, for CAIA-treated $\mathrm{Ccr} 2^{-/-}$DBA1/J mice and control (DBA1/J) mice immunized with a cocktail of collagen type II antibodies (day 0) was examined and a progressive increase in arthritis shown was. At its greatest susceptibility, the clinical score reached a value of 6 at day 10 compared to control mice that which showed no evidence of disease (Figure 1(a)). An examination of the fore and hind paw thicknesses showed no significant difference between the CAIA mice and control animals (Figures 1(b)-1(c)) across days $3-10$, except for a significant difference in the fore paws at day 3 that was not significant at later days.

3.2. Protease Activity Assessment of CAIA. We next hypothesized that the acute inflammatory changes seen in CAIA $\mathrm{Ccr} 2^{-/-}$DBA1/J mice manifested sooner than clinically measurable once (paw thickness) and would only be detectable by in vivo, MMP-activatable fluorescent imaging. In line with our hypothesis, precise in situ quantification of acute inflammation was detected in the arthritic fore and hind paws. We employed protease-targeted fluorescence imaging to look for evidence of acute inflammation in CAIA of Ccr $2^{-/-}$DBA1/J $(n=3)$ and control mice $(n=2)$ (Figure 2). Mice received an intravenous injection of MMPsense 680 (protease-activated fluorescent probe) to directly quantify 


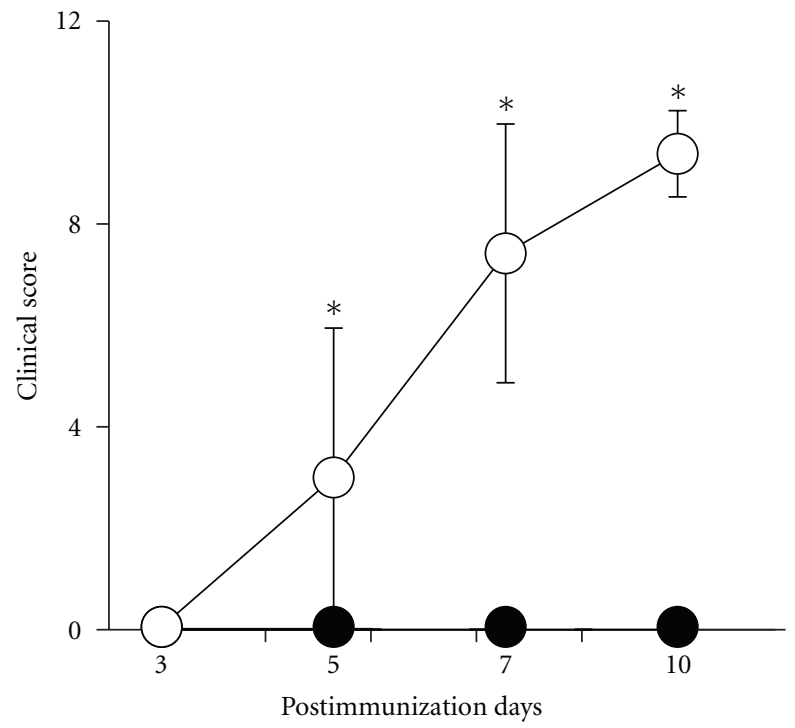

(a)

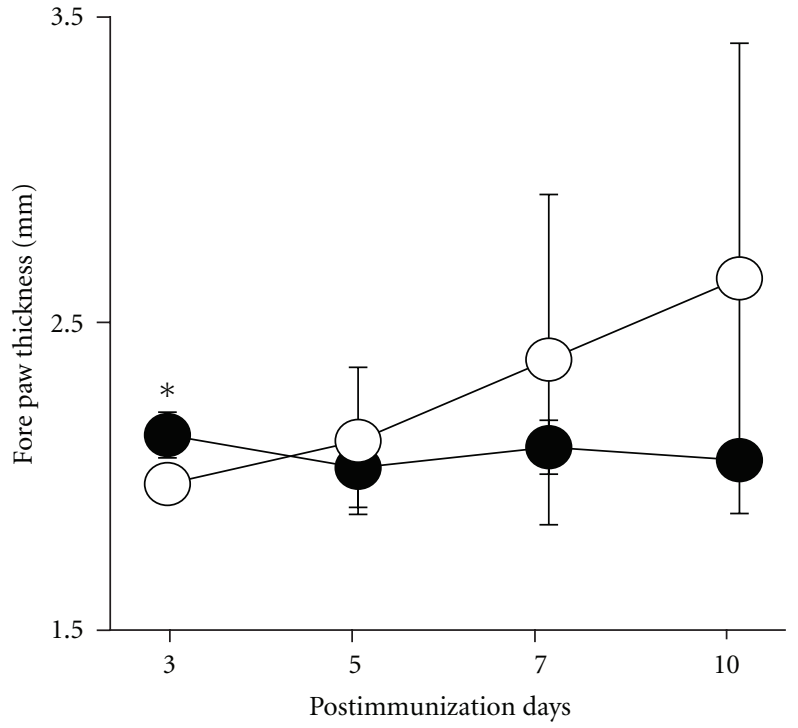

(b)

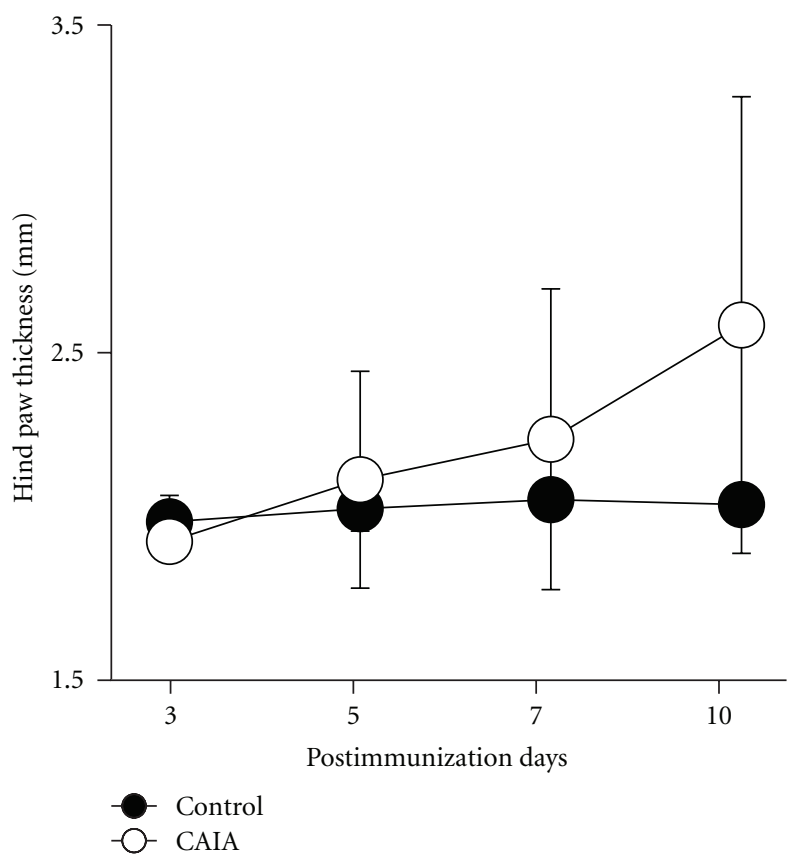

(c)

Figure 1: Clinical assessment of $C \mathrm{cr} 2^{-/-}$DBA1/J animals after receiving CAIA. The development of RA was examined clinically. Mice received immunization intravenously with collagen type II antibody or PBS (day 0) followed by lipopolysaccharide stimulation or with saline for control (day 3). (a) Increased susceptibility to arthritis in mice immunized (day 0 ) with collagen type II antibody (CAIA: $n=3$ ) compared to control mice (PBS: $n=2$ ) without a significant change in fore or hind paw thickness $(\mathrm{b}-\mathrm{c}$ ). Data is represented as the mean \pm SD.

infiltrating neutrophils and macrophages during acute inflammation. A comparison of the mean fluorescence intensity (MFI; photons/second), produced from the local activation of proteases, produced an intense signal in the inflamed synovium in the fore and hind paws of $\mathrm{Ccr}^{-/-} \mathrm{DBA} 1 / \mathrm{J}$ mice but not control mice (Figure 2). An increase in the MFI was seen across days 5,7 , and 10 in CAIA mice (bottom panel) compared to control animals (top panel). For example, acute inflammation in CAIA of $C \mathrm{cr} 2^{-/-}$DBA1/J mice peaked on day 7 at $(4.33 \mathrm{E} \pm 09 \mathrm{MFI})$ and then moved towards resolution of inflammation at day $10(1.92 \mathrm{E} \pm 09 \mathrm{MFI})$. On the other hand, control mice maintained a consistent degree of activation at day $5(0.69 \mathrm{E} \pm 09 \mathrm{MFI})$, day $7(0.59 \mathrm{E} \pm 09 \mathrm{MFI})$, and day $10(0.55 \mathrm{E} \pm 09 \mathrm{MFI})$. Notably, there was an increased fluorescence activity (designated by the asterisk in Figure 2) in the fore and hind paws that was not previously detectable 

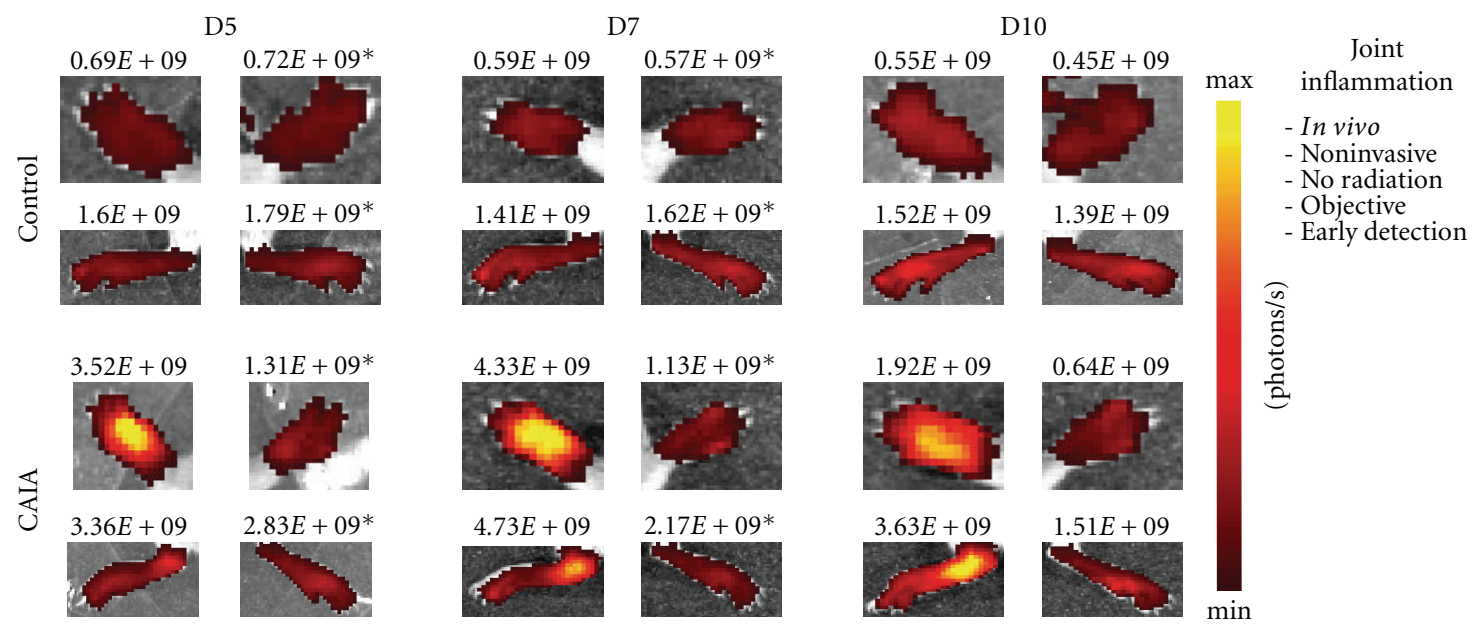

FIGURE 2: In vivo quantification of protease activation in acute arthritis. Comparison of the mean fluorescence intensity in acute inflammation in CAIA mice, in vivo. Hind and fore paw inflammation recruited activated monocytes and neutrophils in Ccr2 ${ }^{-/-}$DBA1/J $(n=3)$ mice. Recruited cells were visualized with MMPsense 680, a molecular probe that fluoresces upon specific cleavage by MMP 2, 3, 9 , and 13, imaged on IVIS spectrum at excitation of $675 \mathrm{~nm}$ and emission of $720 \mathrm{~nm}$. ${ }^{*}$ The right paws and hind feet of the mice increased activity as seen by the values, but the inflammation was not visually apparent. Photons are listed, data presented as mean \pm SD.

by the conventional measures of arthritis (i.e., clinical score, paw thickness, and histology).

\subsection{Histological Assessment of CAIA. To confirm the inflam-} matory changes detected by fluorescence imaging of protease activity, we performed histological analysis on the arthritic ankle joints of $\mathrm{Ccr}^{-/-}$DBA1/J and control mice (Figure 3). In the CAIA model, arthritis resolves by days $10-14$, so we chose postimmunization day 5 to discriminate histological changes in CAIA. The ankle joints were formalin fixed, embedded in paraffin, sectioned for histology, and stained with hemotoxylin and eosin. Arthritis in CAIA of $\mathrm{Ccr}^{-/-}$ DBA1/J mice resulted in the marked elevation of neutrophil infiltrates, bone and cartilage erosion, pannus formation, and fibrin deposition (Figure 3, bottom panel) whereas control animals did not show any of these alterations (Figure 3, top panel). Overall, the acute changes in CAIA mice confirmed the acute inflammation detectable by the in vivo MMP-activatable fluorescent imaging, whereas the control mice showed no significant histological changes.

\section{Discussion}

Our study utilized an MMP-mediated fluorescence imaging agent to detect acute and early inflammation in CAIA. We demonstrated that MMPsense 680 targeted the inflamed synovium in CAIA and that protease activity precisely corresponded to acute inflammation in advance of clinical detection (paw thickness) of disease activity. The use of the protease-activatable imaging agent made it possible to visualize and quantify early inflammatory events (days 5, 7, and 10) outside the conventional methods of measuring disease that rely heavily on subjective assessments. Fluorescent protease imaging proved capable of discriminating acute inflammation in arthritic $\mathrm{Ccr}^{-/-}$DBA1/J mice.
At early stages of arthritis, there appears to be a window of opportunity for when immune dysfunction in RA may potentially be reversible [11]. This window exists at the time prior to the development of histological evidence of pannus and joint damage. However, there does not exist a clinical method of detecting acute events in arthritis with such sensitivity. Much effort has been done to identify biomarkers to detect and possibly quantify the severity of RA disease. Clinically, the most commonly measured markers of inflammation are C-reactive protein (CRP) and ESR [11]. Experimentally, the RF and anticollagen antibodies are the most common measured biomarkers of inflammation. Yet, neither holds sufficient sensitivity to be meaningful for acute stage of disease detection.

To date, current research efforts do not sufficiently measure early events of acute inflammation in arthritis although such knowledge is critical for detecting arthritis early, developing novel intervention that targets early events, and monitoring disease remission. Adding to this problem is the fact that most studies utilizing experimental models of arthritis were limited by the use of standard clinical (clinical scoring/grading and thickness/volume measures) or traditional post mortem (histology) assessments to measure arthritis which were not sensitive enough to detect and quantifiably measure early inflammation over the course of the disease. MMP-mediated monitoring of early arthritis may enable early monitoring of inflammatory events and holds the potential for transforming the interpretation of research efforts in arthritis models.

\section{Conclusion}

Protease-activatable imaging agents are potent tools that afford non invasive, in vivo, in situ, and quantifiable measures of arthritis progression and are specifically sensitive 

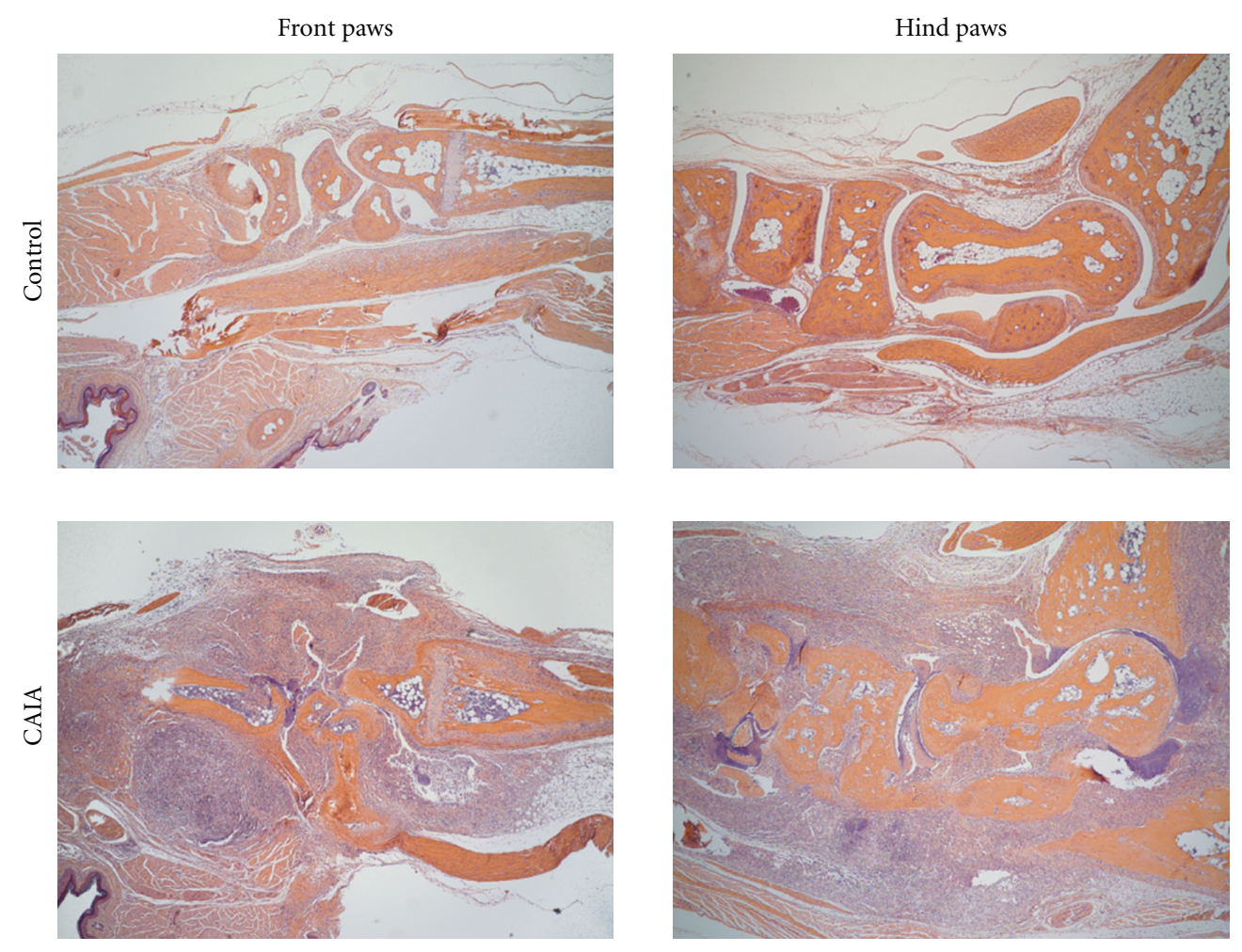

FIGURE 3: Histological findings after CAIA administration. Mice were immunized with collagen type II antibody or PBS (day 0) followed by lipopolysaccharide stimulation or received saline as a control on day 3. On day 5, ankle joints were fixed in formalin, paraffin-embedded, sectioned, and stained with hemotoxylin and eosin. (Top panel) Representative histology with acute inflammation showing increased synovial hyperplasia, bone and cartilage destruction, pannus invasion, and fibrin deposition (bottom panel) in Ccr $2^{-/-}$DBA1/J mice, compared to no acute inflammation in control mice treated with PBS at day 0 and saline at day 3 (bottom panel). Results are representative of independent experiments with 2-3 mice/group.

enough to detect early stages of disease. While the imaging technology is not applicable for clinical use, it does afford a powerful tool for assessing models of RA to gain novel spatial and temporal insights into arthritis disease progression, hence enhancing the understanding of molecular and cellular mechanisms underlying RA with the potential for expanding assessments for treatments.

\section{Abberviation}

MMP: Matrix metalloproteinase

DC: Dendritic cells

RA: Rheumatoid arthritis

CAIA: Collagen antibody induced arthritis

LPS: Lipopolysaccharide.

\section{Acknowledgments}

This work was supported in part by the National Institutes of Health (NIH) grants (AI48644 and AR052755) and VA Merit to S.S.A. J. M. Ibarra and F. Jimenez contributed equally to this work.

\section{References}

[1] R. C. Lawrence, C. G. Helmick, F. C. Arnett et al., "Estimates of the prevalence of arthritis and selected musculoskeletal disorders in the United States," Arthritis and Rheumatism, vol. 41, no. 5, pp. 778-799, 1998.

[2] C. H. MacLean, R. Louie, B. Leake et al., "Quality of care for patients with rheumatoid arthritis," Journal of the American Medical Association, vol. 284, no. 8, pp. 984-992, 2000.

[3] O. Sangha, "Epidemiology of rheumatic diseases," Rheumatology, vol. 39, no. 2, pp. 3-12, 2000.

[4] F. M. Brennan, A. Jackson, D. Chantry, R. Maini, and M. Feldmann, "Inhibitory effect of TNF $\alpha$ antibodies on synovial cell interleukin-1 production in rheumatoid arthritis," Lancet, vol. 2, no. 8657, pp. 244-247, 1989.

[5] W. P. Arend and J. M. Dayer, "Inhibition of the production and effects of interleukin-1 and tumor necrosis factor $\alpha$ in rheumatoid arthritis," Arthritis and Rheumatism, vol. 38, no. 2, pp. 151-160, 1995.

[6] M. P. Quinones, S. K. Ahuja, F. Jimenez et al., "Experimental arthritis in CC chemokine receptor 2-null mice closely mimics severe human rheumatoid arthritis," Journal of Clinical Investigation, vol. 113, no. 6, pp. 856-866, 2004.

[7] M. P. Quinones, C. A. Estrada, Y. Kalkonde et al., "The complex role of the chemokine receptor CCR2 in collagen-induced arthritis: implications for therapeutic targeting of CCR2 in 
rheumatoid arthritis," Journal of Molecular Medicine, vol. 83, no. 9, pp. 672-681, 2005.

[8] J. M. R. Frade, M. Mellado, G. Del Real, J. C. Gutierrez-Ramos, P. Lind, and C. Martinez-A. C., "Characterization of the CCR2 chemokine receptor: functional CCR2 receptor expression in B cells," Journal of Immunology, vol. 159, no. 11, pp. 55765584, 1997.

[9] A. Katrib, P. P. Tak, J. V. Bertouch et al., "Expression of chemokines and matrix metalloproteinases in early rheumatoid arthritis," Rheumatology, vol. 40, no. 9, pp. 988-994, 2001.

[10] A. B. Blom, P. L. Van Lent, S. Libregts et al., "Crucial role of macrophages in matrix metalloproteinase-mediated cartilage destruction during experimental osteoarthritis: involvement of matrix metalloproteinase 3," Arthritis and Rheumatism, vol. 56, no. 1, pp. 147-157, 2007.

[11] J. Dale and D. Porter, "Pharmacotherapy: concepts of pathogenesis and emerging treatments. Optimising the strategy of care in early rheumatoid arthritis," Best Practice \& Research Clinical Rheumatology, vol. 24, no. 4, pp. 443-455, 2010. 


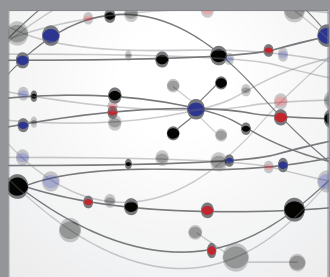

The Scientific World Journal
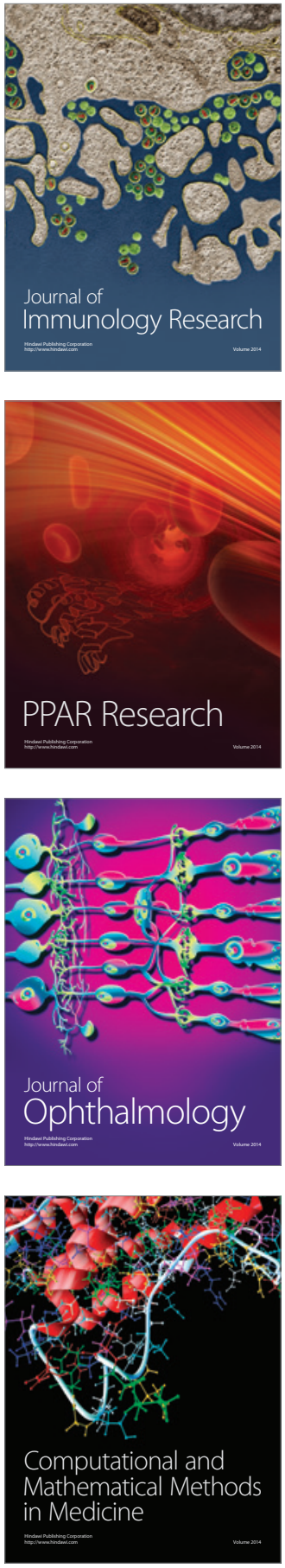

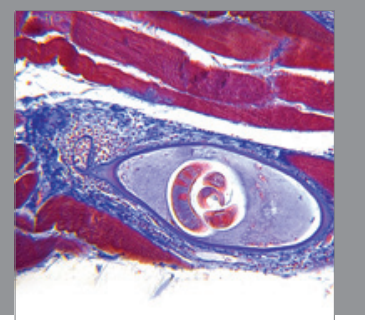

Gastroenterology

Research and Practice
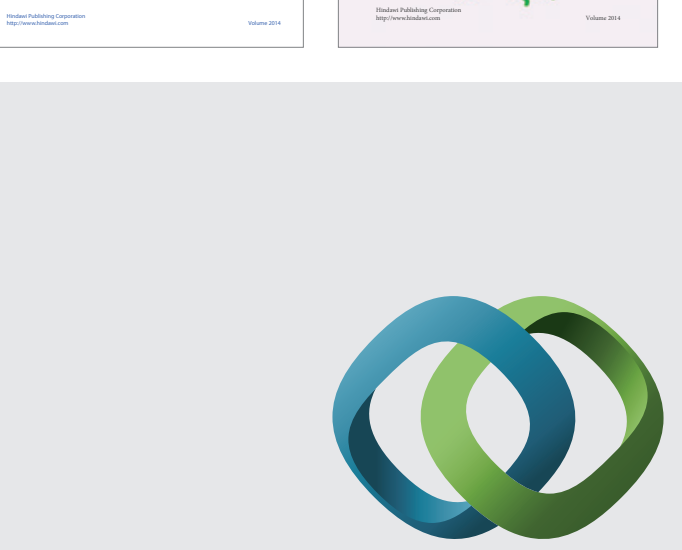

\section{Hindawi}

Submit your manuscripts at

http://www.hindawi.com
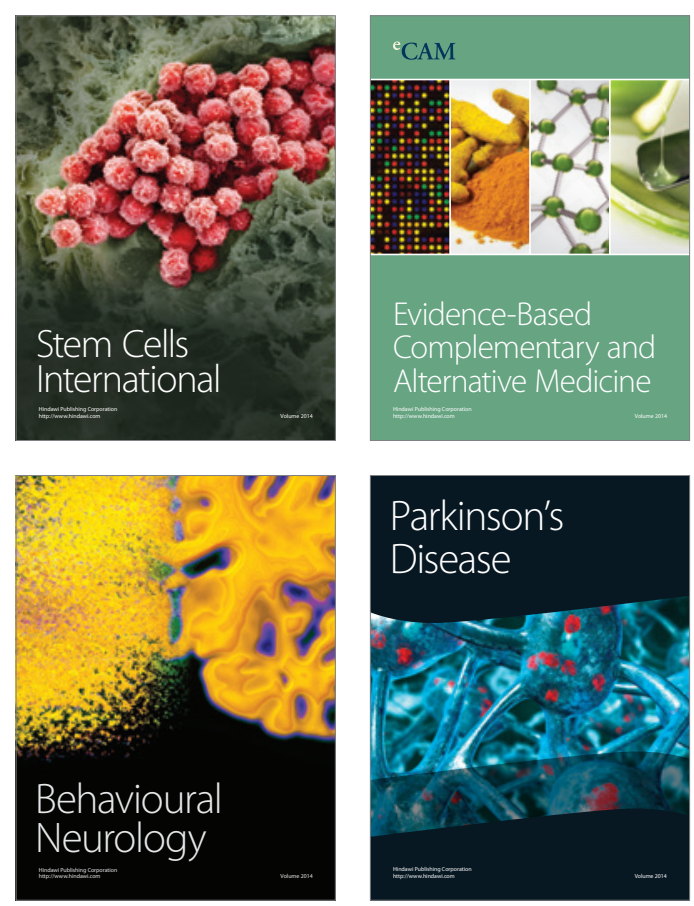

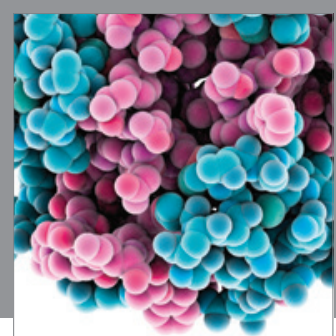

Journal of
Diabetes Research

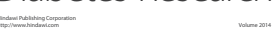

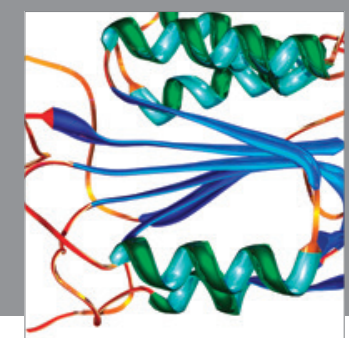

Disease Markers
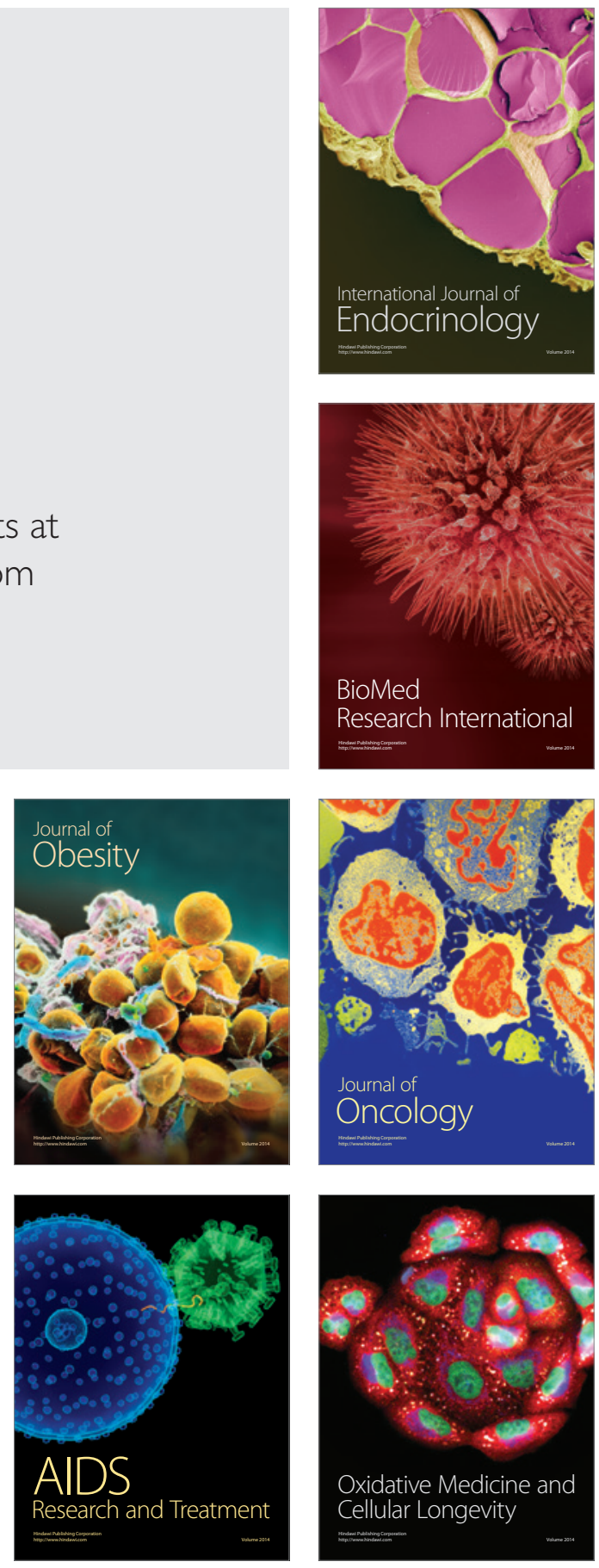